\title{
Cross-correlations in a quantum dot Cooper pair splitter with ferromagnetic leads
}

\author{
Piotr Trocha ${ }^{1, *}$ and Kacper Wrześniewski ${ }^{1}$ \\ ${ }^{1}$ Faculty of Physics, Adam Mickiewicz University, 61-614 Poznań, Poland
}

(Dated: July 25, 2018)

\begin{abstract}
We investigate Andreev transport through a quantum dot attached to two external ferromagnetic leads and one superconducting electrode. The transport properties of the system are studied by means of the real-time diagrammatic technique in the sequential tunneling regime. To distinguish various contributions to Andreev current we calculate the current cross-correlations, i.e., correlations between currents flowing through two junctions with normal leads. We analyze dependence of current cross-correlations on various parameters of the considered model, both in linear and nonlinear transport regimes. The processes and mechanisms leading to enhancement, suppression or sign change of current cross-correlations are examined and discussed. Interestingly, our results show that for specific transport regimes splitted Cooper pair results in two uncorrelated electrons. However, utilizing ferromagnetic leads instead of non-magnetic electrodes can result in positive current crosscorrelations.
\end{abstract}

\section{INTRODUCTION}

Electronic transport phenomena in hybrid quantum dot (QD) systems have recently attracted great attention $^{1-12}$. Particularly, QD systems with one superconducting lead and two normal metal electrodes enable creation of nonlocal entangled electron pairs ${ }^{13-17}$. Moreover, splitting of Cooper pairs into two spatially separated electrodes has been demonstrated experimentally in a carbon nanotube double quantum dot system ${ }^{18-20}$. These investigations are important both from the fundamental point of view and also for future applications in quantum computing $^{21}$.

When the applied bias voltage window is in the superconducting gap, the current flows mainly due to Andreev reflection processes ${ }^{22}$ while the quasiparticle tunneling becomes negligible in the low temperature limit. Generally, in-gap tunneling processes can occur via direct Andreev reflection (DAR), crossed Andreev reflection (CAR), or elastic cotunneling (EC). Under certain conditions, by properly changing device parameters, one can tune the contributions due to CAR and DAR, or EC processes or even suppress one of them. Thus, a tool which allows to distinguish these contributions is sought. An important one seems to be given by current crosscorrelations, i.e., correlations calculated between currents flowing through two junctions with normal leads ${ }^{23,24}$.

The current cross-correlations can deliver a deeper insight into tunneling processes contributing to electronic transport $^{25}$ and has been reported experimentally ${ }^{26-28}$ in various setups. Generally, positive current crosscorrelations can be attributed with interactions supporting currents in both junctions. Especially, they are present in systems with superconducting electrodes ${ }^{29-38}$, like Cooper pair splitters, in which enhancement of positive current cross-correlations can be associated with high Cooper pair splitting efficiency. On the other hand, positive current cross-correlations can be suppressed by interactions, which mutually block the currents flowing through two junctions. The tunneling processes that occur in opposite directions are associated with negative sign of current cross-correlations.

Interestingly, negative current cross-correlations in electron beam splitter device have been observed experimentally in a Hanbury Brown-Twiss setup ${ }^{39,40}$. Moreover, in a paramagnetic multiterminal quantum dot current cross-correlations have been found to be negative ${ }^{41}$. However, in a three-terminal QD system coupled to ferromagnetic leads some positive cross-correlations between output currents can appear as a result of dynamical spin blockade on the dot not observed in the paramagnetic case $^{42}$.

The current cross-correlations are also useful for determining the efficiency and fidelity of an electron-entangler device and allow to formulate Bell-type inequalities ${ }^{43,44}$. Their violation provides an evidence of nonlocality of split pairs of electrons.

One should also bear in mind that positive current cross-correlations noticed in hybrid superconducting structures are not always due to CAR processes ${ }^{33,45}$. For instance, such situation has been predicted for normal metal-superconductor-normal metal hybrid structures with highly transparent interfaces ${ }^{45}$.

In hybrid QD's systems being in proximity to superconductor the formation of entangled electrons' pairs seems to be rather natural. Therefore, superconducting lead acts as a source of entangled pairs of electrons as its ground state is occupied by Cooper pairs in a spin-singlet state. Furthermore, these Cooper pairs can be extracted from a superconductor by tunneling through the dot's states into the normal metal leads. However, two Andreev tunneling processes have to be distinguished: direct Andreev reflection (DAR) and crossed Andreev reflection (CAR). The former process occurs when Cooper pair extracted from superconductor is transmitted to the same normal lead, whereas in the latter one, entangled pair of electrons leaving the superconductor is split into its individual electrons which end in two spatially-separated leads. A minimal condition to assure the split electrons stay correlated is that the coherence length of Cooper pair has to exceed the width of the superconducting source contact. In an efficient electron entangler, which 
converts a charge current to a flux of spin-entangled electron pairs, the CAR processes have to be enforced. By analyzing current cross correlations one is able to distinguish regions in device's parameters space with high and low Cooper pairs splitting efficiency. Particularly, large positive current cross-correlations can be associated with enhancement in Cooper pair splitting efficiency, while small values of the aforementioned quantity indicate low splitter's efficiency.

In this paper we study dependence of current crosscorrelations on various parameters of the considered model focusing on the in-gap transport regime. The processes and mechanisms leading to enhancement or suppression of current cross-correlations are examined and discussed. Moreover, the influence of external magnetic field and ferromagnetism of external leads on current cross-correlations is also investigated. We use the realtime diagrammatic technique to calculate transport characteristics. In general, the external weakly coupled leads are assumed to be metallic ferromagnetic electrodes. We consider collinear magnetic configurations, i.e. when magnetic moments of both leads are aligned in the same directions (parallel) or oppositely (antiparallel).

The paper is organized in the following way: In Section II we introduce the model of the system and present theoretical background. This includes description of the model and also description of the method used to calculate quantities of interest. In Section III numerical results are presented and discussed. In this section we distinguish the cases of leads with and without spin polarization. Additionally, the cases of system with and without external magnetic field are considered separately. Finally, Section IV includes brief summary and general conclusions.

\section{THEORETICAL DESCRIPTION}

The system taken into considerations consists of singlelevel quantum dot attached to two normal metal and one superconducting lead as shown in Fig. 1.

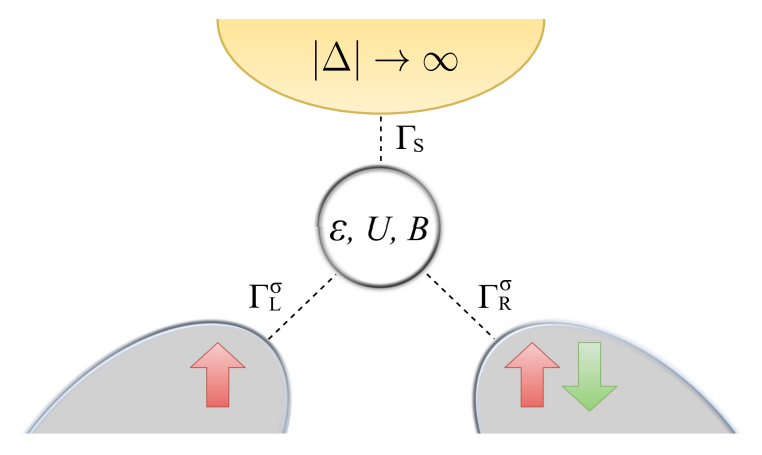

FIG. 1: Schematic picture of the QD system coupled to two normal metal and one superconducting leads.
The Hamiltonian of the system acquires the following form:

$$
H=\sum_{\beta=L, R} H_{\beta}+H_{S}+H_{Q D}+H_{T},
$$

where the first term, $H_{\beta}$ for $\beta=L, R$, describes the left $(L)$ and right $(R)$ electrodes in the noninteracting quasiparticle approximation, $H_{\beta}=\sum_{\mathbf{k} \sigma} \varepsilon_{\mathbf{k} \beta \sigma} c_{\mathbf{k} \beta \sigma}^{\dagger} c_{\mathbf{k} \beta \sigma}$ with $\varepsilon_{\mathbf{k} \beta \sigma}$ denoting the single particle energy.

The second term in Eq. (1) describes the s-wave BCS superconducting lead in the mean field approximation

$$
\begin{aligned}
H_{S} & =\sum_{\mathbf{k}, \sigma} \varepsilon_{\mathbf{k} S} c_{\mathbf{k} S}^{\dagger} c_{\mathbf{k} S} \\
& +\sum_{\mathbf{k}}\left(\Delta^{*} c_{\mathbf{k} S \downarrow} c_{-\mathbf{k} S \uparrow}+\Delta c_{-\mathbf{k} S \uparrow}^{\dagger} c_{\mathbf{k} S \downarrow}^{\dagger}\right),
\end{aligned}
$$

with $\varepsilon_{\mathbf{k} S}$ denoting the relevant single-particle energy and $\Delta$, assumed real and positive, standing for the order parameter of the superconductor.

The third term of Hamiltonian describes single-level quantum dot and acquires the following form:

$$
H_{Q D}=\sum_{\sigma} \varepsilon_{\sigma} d_{\sigma}^{\dagger} d_{\sigma}+U n_{\uparrow} n_{\downarrow}
$$

where $\varepsilon_{\sigma}$ and $U$ denote the spin dependent dot's energy level and the corresponding Coulomb integral. Applying external magnetic field dot's energy level becomes split, $\varepsilon_{\sigma}=\varepsilon \pm B / 2$ with $B$ denoting Zeeman splitting energy.

Finally, tunneling of electrons between the leads $(L, R, S)$ and the quantum dot can be modelled by the Hamiltonian

$$
H_{T}=\sum_{\mathbf{k} \sigma} \sum_{\beta=L, R, S}\left(V_{\mathbf{k} \sigma}^{\beta} c_{\mathbf{k} \beta \sigma}^{\dagger} d_{\sigma}+\text { h.c. }\right)
$$

with $V_{\mathbf{k} \sigma}^{\beta}$ denoting the relevant tunneling matrix elements.

In the wide band approximation dot's coupling to the normal metal electrodes can be assumed to be energy independent and constant, $\Gamma_{L}^{\sigma}=\Gamma_{L}(1+\tilde{\sigma} p)$, and $\Gamma_{R}^{\sigma}=\Gamma_{R}(1+\eta \tilde{\sigma} p)$ with $\tilde{\sigma}=1$ for $\sigma=\uparrow$ and $\tilde{\sigma}=-1$ for $\sigma=\downarrow$. Here, $p$ denotes the spin polarization of magnetic leads assumed the same for the left and right electrodes, whereas $\eta= \pm 1$ for parallel (upper sign) and antiparallel (lower sign) magnetic alignment of ferromagnetic leads. Furthermore, we assume symmetric couplings, $\Gamma_{L}=\Gamma_{R} \equiv \Gamma / 2$.

As we are interested in Andreev transport regime we can take the limit of an infinite superconducting gap, $\Delta \rightarrow \infty$. Then the quantum dot coupled to the superconducting lead is described by an effective Hamiltonian ${ }^{46}$ :

$$
H_{Q D}^{\mathrm{eff}}=\sum_{\sigma} \varepsilon_{\sigma} d_{\sigma}^{\dagger} d_{\sigma}+U n_{\uparrow} n_{\downarrow}-\frac{\Gamma_{S}}{2}\left(d_{\uparrow}^{\dagger} d_{\downarrow}^{\dagger}+d_{\downarrow} d_{\uparrow}\right)
$$


where the effective pair potential $\Gamma_{S}$ is the coupling strength between the dot and superconducting electrode and acquires the form $\Gamma_{S}=2 \pi\left|V^{S}\right|^{2} \rho_{S}$ with $\rho_{S}$ denoting BCS density of states in the normal state.

The eigenstates of the effective dot's Hamiltonian acquire the form: $|\sigma\rangle$, and $| \pm\rangle=$ $1 / \sqrt{2}\left(\sqrt{1 \mp \delta /\left(2 \varepsilon_{A}\right)}|0\rangle \mp \sqrt{1 \pm \delta /\left(2 \varepsilon_{A}\right)}|2\rangle\right), \quad$ while the corresponding eigen-energies are: $E_{\uparrow}=\varepsilon_{\uparrow}, E_{\downarrow}=\varepsilon_{\downarrow}$, and $E_{ \pm}=\delta / 2 \pm \varepsilon_{A}$, with $\delta=\varepsilon_{\uparrow}+\varepsilon_{\downarrow}+U$. Here, $\varepsilon_{A}=\sqrt{\delta^{2}+\Gamma_{S}^{2}} / 2$ measures the energy difference between the states $|+\rangle$ and $|-\rangle$.

The Andreev bound states' energies are defined as:

$$
E_{\alpha, \beta}^{A}=\alpha \frac{U}{2}+\frac{\beta}{2} \sqrt{\delta^{2}+\Gamma_{S}^{2}},
$$

where $\alpha, \beta= \pm$. These energies are the excitation energies of the dot decoupled from the normal metal leads.

In order to determine the transport properties of the system we employ the real-time diagrammatic technique $^{47-49}$. The stationary occupation probability $p_{\chi}^{s t}$ of a state $|\chi\rangle$ can be found from:

$$
\mathbf{W} \mathbf{p}^{\mathbf{s t}}=0,
$$

where $\mathbf{p}^{\text {st }}$ is the vector containing probabilities $p_{\chi}^{s t}$ and the elements $W_{\chi \chi^{\prime}}$ of self-energy matrix $\mathbf{W}$ account for transitions between the states $|\chi\rangle$ and $\left|\chi^{\prime}\right\rangle$.

The current flowing through the junction with lead $\alpha=$ $(L, R)$ can be found from:

$$
I_{\alpha}=\frac{e}{2 \hbar} \operatorname{Tr}\left\{\mathbf{W}^{I_{\alpha}} \mathbf{p}^{s t}\right\},
$$

where the self-energy matrix $\mathbf{W}^{I_{\alpha}}$ is similar to $\mathbf{W}$, but it takes into account the number of electrons transferred through a given junction.

The current cross-correlations in the sequential tunneling approximation, $S_{L R}$, are defined $\operatorname{as}^{48}$ :

$$
S_{L R}=\frac{e^{2}}{\hbar} \operatorname{Tr}\left\{\left[\mathbf{W}^{I_{L}} \mathbf{P} \mathbf{W}^{I_{R}}+\mathbf{W}^{I_{R}} \mathbf{P} \mathbf{W}^{I_{L}}\right] \mathbf{p}^{s t}\right\},
$$

where the propagator $\mathbf{P}$ is determined from $\mathbf{W P}=$ $\mathbf{p}^{s t} \mathbf{e}^{T}-\mathbf{1}$, with $\mathbf{e}^{T}=(1,1, \ldots, 1)$.

\section{NUMERICAL RESULTS}

We present the numerical results for Andreev transport assuming large superconducting-gap limit. Here, we show the differential conductance associated with current injected/extracted into/from superconductor and the corresponding current cross-correlations. The differential conductance, $G_{S}$, is calculated as $G_{S}=d I_{S} / d V$ with $I_{S}$ denoting Andreev current. The Andreev current is simply obtained from Kirchoff's law as $I_{S}=I_{L}+I_{R}$.

The section is divided into two parts. In the first part of the section the case of nonmagnetic external electrodes $(p=0)$ is considered, whereas in the second part we investigate the case of magnetic leads $(p \neq 0)$. Moreover, throughout the sections we consider two distinct cases, with and without external magnetic field leading to finite or no Zeeman splitting.

\section{A. Nonmagnetic leads $(p=0)$}

In this section the transport properties of QD coupled to one superconducting electrode and two nonmagnetic metallic leads are considered. The differential conductance and corresponding current cross-correlations are calculated for two cases. The former case corresponds to situation when no external magnetic field is applied, whereas in the latter one the influence of magnetic field is taken into account.

\section{No external magnetic field, $B=0$}

The differential conductance $G_{S}$ corresponding to Andreev current and the respective current crosscorrelations $S_{L R}$ calculated for zero magnetic field, $B=$ 0 , are shown in Fig. 2 as a function of bias voltage applied to the two normal leads. The system is biased in the following way: $\mu_{L}=\mu_{R}=e V$ and grounding the superconducting electrode $\mu_{S}=0$. We introduce detuning parameter, $\delta=2 \varepsilon+U$, for spin-degenerate dot's level, i. e. for $\varepsilon_{\uparrow}=\varepsilon_{\downarrow} \equiv \varepsilon$.

First of all, one can notice that differential conductance obeys the symmetry, $G_{S}(\delta, e V)=G_{S}(-\delta,-e V)$. Due to the fact that the Andreev current (not shown) is optimized when particle-hole symmetry holds, it becomes significant only for small detuning $\delta$. As a result, $G_{S}$ acquires the largest values for $\delta$ close to zero. The differential conductance, $G_{S}$, reveals a peak each time the electrochemical potential of normal metal leads crosses one of the Andreev levels. Generally, for zero magnetic field, $G_{S}$ exhibits four peaks associated with four Andreev levels. However, for $|\delta|=\sqrt{U^{2}-\Gamma_{S}^{2}}$ only three peaks appear as the states $E_{A}^{+-}$and $E_{A}^{-+}$become degenerate. Moreover, $G_{S}$ is asymmetric with respect to the bias reversal for finite detuning $\delta \neq 0$, which is directly related with the behavior of the Andreev current.

In Fig. 2(b) the corresponding current crosscorrelations, $S_{L R}$, are shown. One can notice that $S_{L R}$ vanishes in the Coulomb blockade regime when the dot is occupied by single electron, i.e., for $E_{+-}<e V<E_{-+}$ and for $|\delta|<\sqrt{U^{2}-\Gamma_{S}^{2}}$. This is the result of vanishing current in the Coulomb blockade regime as two electrons are required to form Cooper pair. The current crosscorrelations also vanish for $|\delta|>\sqrt{U^{2}-\Gamma_{S}^{2}}$ and for small bias voltage, $E_{A}^{+-}<e V<E_{A}^{-+}$, when no current flows. These regions corresponds to empty or doubly occupied dot regime. For most regions, where the current can flow, $S_{L R}$ acquires positive values, which indicates that CAR 

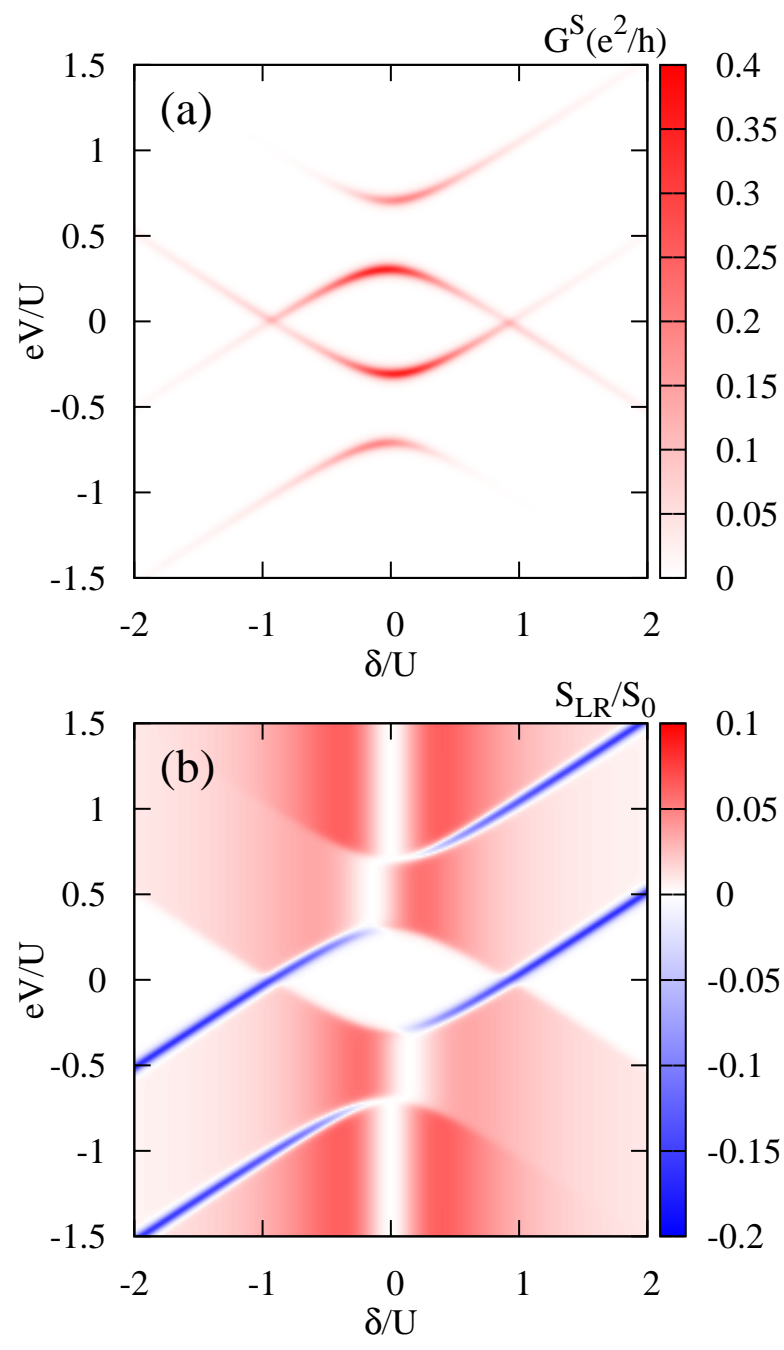

FIG. 2: (color online) Differential conductance $G_{S}$ (a) and corresponding current cross-correlations (b) as a function of bias voltage and detuning $\delta$ calculated for magnetic field, $B=$ 0 . The other parameters are: $U=1$ (used as energy unit), $\Gamma_{S}=0.4, \Gamma=0.01$ and $T=0.015$, with $S_{0}=e^{2} \Gamma / \hbar$.

processes give main contribution to the current. Interestingly, $S_{L R}$ exhibits also negative values. Specifically, $S_{L R}$ becomes negative when bias voltage passes through $E_{-+}^{A}$ for $\delta \gtrsim 0$ and for $\delta \lesssim 0$ when $e V \approx E_{+-}^{A}$. Another two regions of negative current cross-correlations appear for $\delta>0$ when $e V \approx E_{++}^{A}$ and for $\delta<0$ when $e V \approx E_{--}^{A}$. This happens each time the bias voltage $e V$ approaches dot's level $\varepsilon$ or $\varepsilon+U$. Then, single electron can tunnel from the left lead onto the dot and re-tunnel into the right lead. Reverse tunneling processes $(R \rightarrow L)$ occur with the same probability. Hence, there is no net charge current, but these processes give contribution to the current cross-correlation, specifically to its negative values, indicating that tunneling processes by left and right junctions occur in opposite directions and compensate each other.

It is worth noting that $S_{L R}$ vanishes in the vicinity of particle-hole symmetry point $(\delta \approx 0)$ for $\mathrm{eV}>E_{++}^{A}$ and for $e V<E_{--}^{A}$, irrespective of the presence of magnetic field. However, it can not be stated that Andreev current flows only due to DAR processes although $S_{L R}$ vanishes. According to symmetry of the system, both DAR and CAR processes equally contribute to the Andreev transport. It is enough to consider positive bias case with $e V>E_{++}^{A}$ as for negative bias voltage with $e V<E_{--}^{A}$ an analogous discussion can be applied. Due to strong coupling between the dot and superconducting lead $\left(\Gamma_{S} \gg \Gamma\right)$ fast coherent oscillations of Cooper pairs occur between $Q D$ and superconductor. These coherent oscillations are occasionally interrupted by tunneling of single electron from normal lead (left or right). Average interval of time between tunneling of an electron from normal lead into the dot is relatively large $(2 \hbar / \Gamma)$ comparing with the Cooper pairs oscillations' pe$\operatorname{riod}\left(2 \hbar / \Gamma_{S}\right)$. Thus, from the point of view of normal leads, single electrons tunnel into the dot independently (of the oscillations) ${ }^{50}$. Thus, such uncorrelated singleelectron tunneling events of two subsequent electrons originating from different normal leads can not give impact to $S_{L R}$. To support the above explanation let us present more formal considerations. The zero-frequency cross correlations between the currents flowing through the left and right junctions can be defined as follows:

$$
S_{L R}=\int_{-\infty}^{\infty} d t\left\langle\delta I_{L}(t) \delta I_{R}(0)+\delta I_{R}(0) \delta I_{L}(t)\right\rangle
$$

with $\delta I_{\alpha}(t)=\hat{I}_{\alpha}(t)-\left\langle\hat{I}_{\alpha}\right\rangle$ and $\hat{I}_{\alpha}$ being the current operator.

For the bias voltages $e V>E_{++}^{A}$ and for $e V<E_{--}^{A}$ in the particle-hole symmetry point $(\delta / U=0)$ the Andreev current is maximized, but simultaneously the current cross-correlations are completely suppressed, i. e. $S_{L R}=0$. This behavior can be explained by analysing the states' probabilities of the quantum dot and matrix elements of current matrices $W^{\mathrm{I}_{\alpha}}$ for discussed transport parameters. The probability of occupation of each available states, i.e. $|+\rangle,|-\rangle,|\uparrow\rangle$ and $|\downarrow\rangle$ is the same and equals to $p_{\chi}=1 / 4$ with $\chi=+,-, \uparrow, \downarrow$. Moreover, all non-zero matrix $W^{\mathrm{I}_{\alpha}}$ elements for both left and right junctions are equal as well, which means that all possible tunneling events have equal and maximal rates, with left/right junction indifference. This results in a constant average current in each (left/right) junction of equal value. Moreover, it turns out that the currents through both junctions are constant in time. This has been checked by solving rate equation $\dot{\mathbf{p}}(t)=\mathbf{W} \mathbf{p}(t)$ and calculating time evolution of currents through left and right junction. As a result, there are no fluctuations of the current away from the average value, i.e. $\delta I_{\alpha}(t)=\hat{I}_{\alpha}(t)-\left\langle\hat{I}_{\alpha}\right\rangle=0$, and thus, $S_{L R}=0$.

Similar considerations can be applied for bias voltage $E_{-+}^{A}<e V<E_{++}^{A}$ as well as for $E_{--}^{A}<e V<E_{+-}^{A}$. However, now the zero current cross-correlations are realized for slightly shifted $\delta$ from particle-hole symmetry 
point. In the former case, $S_{L R}=0$ is noticed around $\delta=-\frac{\sqrt{2}}{4} \Gamma_{S}$, whereas for the latter one, current crosscorrelations vanish for $\delta=\frac{\sqrt{2}}{4} \Gamma_{S}$.

\section{Finite external magnetic field, $B \neq 0$}

Applying finite magnetic field leads to the splitting of the Andreev bound states and to doubling of corresponding excitation energies. Now, Andreev excitation energies acquire the following form, $E_{\alpha, \beta, \gamma}^{A}=E_{\alpha, \beta}^{A}+\gamma \frac{B}{2}$ with $\gamma= \pm 1$. As a result, more peaks appear in the differential conductance, see e.g. Fig. 3(a) calculated for $B / U=0.5$. One can notice that the range of Coulomb blockade becomes enlarged, as filling the dot with second electron cost the energy $\varepsilon_{\uparrow}+U$.

In the case of finite magnetic field the current crosscorrelations reveal rather minor qualitative and quantitative differences disregarding the effect due to Andreev bound states splitting. First of all, the maximal positive values of current cross-correlations are of the same order as these corresponding to zero magnetic field case. In turn, the negative values of current crosscorrelations become slightly increased. Due to additional Andreev bound states originating from Zeeman splitting $S_{L R}$ exhibits more steps. However, the number of regions where $S_{L R}$ is negative becomes independent of magnetic field. In the remaining regions between the steps $S_{L R}$ vanishes instead of acquiring negative values. The most striking qualitative difference in $S_{L R}$ generated by magnetic field is associated an enhancement of positive current cross-correlations when $\mathrm{eV}$ crosses through $E_{+-+}^{A}\left(E_{-+-}^{A}\right)$ for $\delta \in\left(-\sqrt{B^{2}-\Gamma_{S}^{2}}, \sqrt{U^{2}-\Gamma_{S}^{2}}\right)(\delta \in$ $\left.\left(-\sqrt{U^{2}-\Gamma_{S}^{2}}, \sqrt{B^{2}-\Gamma_{S}^{2}},\right)\right)$, i.e. for bias voltages for which QD passes from singly occupied state to doubly or empty state, respectively, allowing for CAR processes to occur. The widths of these peaks in $S_{L R}$ becomes smaller with decreasing temperature and disappear in the limit $T \rightarrow 0$.

\section{B. Magnetic leads $(p>0)$}

So far, the calculations have been performed for QD Cooper pair beam splitters with nonmagnetic leads. However, because using ferromagnetic leads can be important to estimate entanglement between split electrons $^{51}$, here, we provide comprehensive study of transport properties of the QD Cooper beam splitters with ferromagnetic contacts.

In this section, the effects arising due to magnetism of external weakly coupled leads are considered. Now, the left and right electrodes are spin polarized and the strength of the external leads' polarization is described by spin polarization factor, $p$, assumed equal for both ferromagnetic electrodes. Here, we consider collinear magnetic configurations of ferromagnetic leads: parallel $(\mathrm{P})$

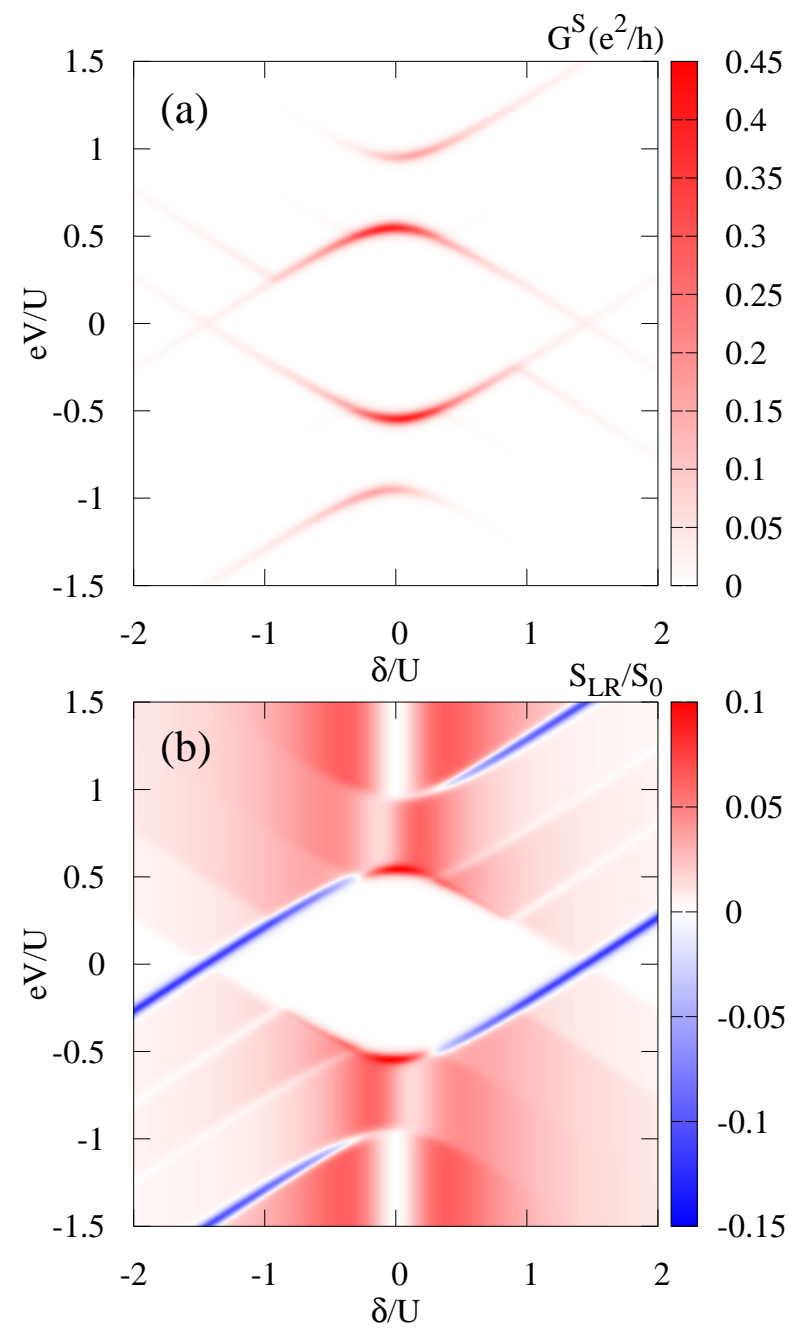

FIG. 3: (color online) Differential conductance $G_{S}$ (a) and corresponding current cross-correlations (b) as a function of bias voltage and detuning $\delta$ calculated for magnetic field, $B / U=0.5$. The other parameters are as in Fig. 2 .

and antiparallel (AP). The former one corresponds to the situation when magnetic moments of ferromagnetic leads are aligned in the same direction, whereas in the latter one they align in the opposite directions.

Let us first consider parallel magnetic configuration. In this case the differential conductance [Fig. 4(a)] reveals several differences comparing with nonmagnetic situation [Fig. 2(a)]. One can notice that maximal intensity of differential conductance becomes suppressed as the minority carriers determine the Andreev current. Accordingly, in the parallel magnetic configuration the density of states of minority carriers rules the rate of injecting/extracting electron pairs, which becomes the bottleneck for the Andreev transport. However, more striking difference is appearance of negative values in the differential conductance. Specifically, negative values of the differential conductance emerge for $|\delta|>\sqrt{U^{2}-\Gamma_{S}^{2}}$ in the vicinity of Andreev levels $E_{-+}^{A}\left(E_{+-}^{A}\right)$ for positive 

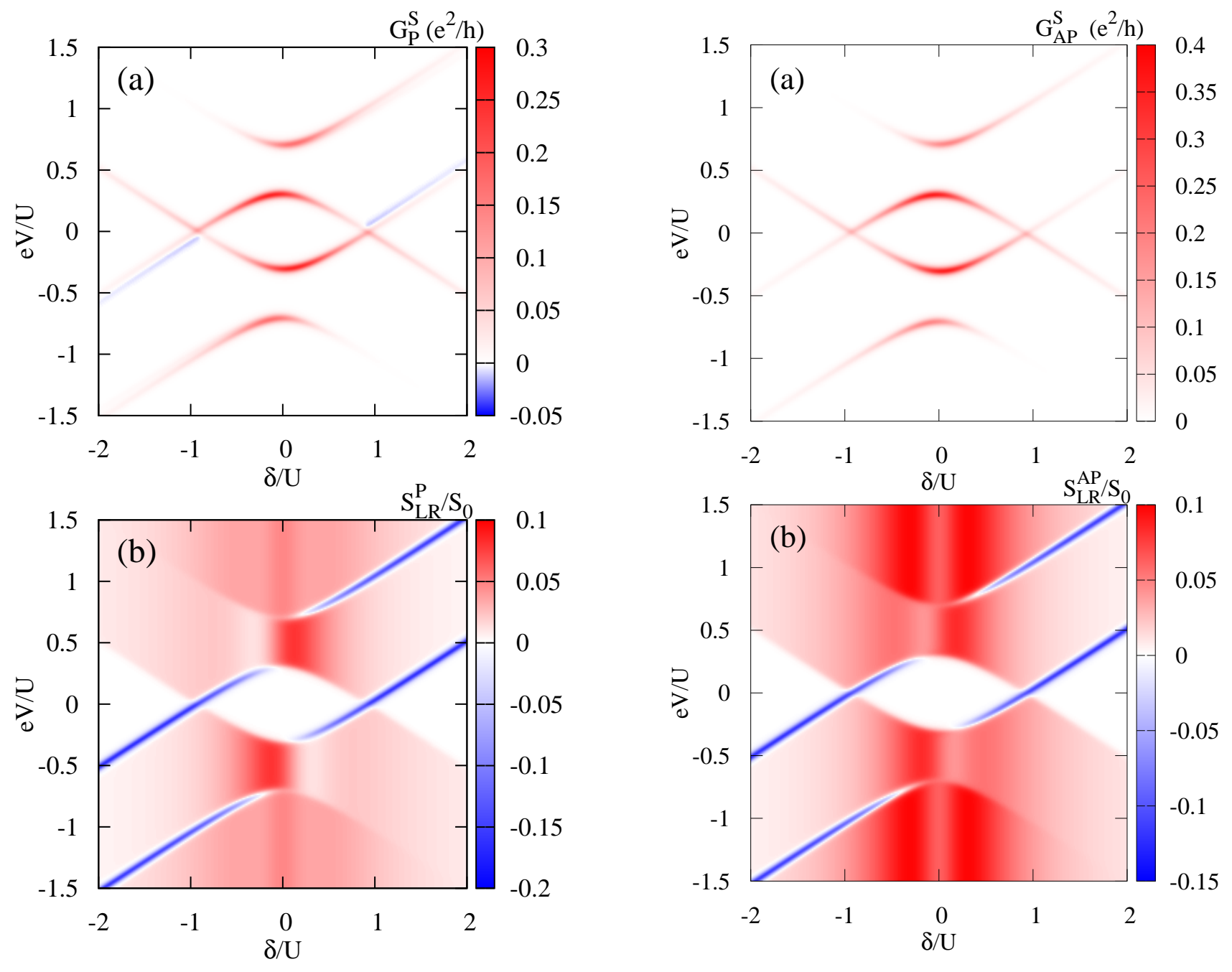

FIG. 4: (color online) Differential conductance $G_{S}$ (a) and corresponding current cross-correlations (b) as a function of bias voltage and detuning $\delta$ in parallel magnetic configuration $\mathrm{P}$ calculated for spin polarization $p=0.5$. The other parameters are as in Fig. 2.

(negative) electrochemical potential shift, see Fig. 4(a). The physical mechanism leading to negative differential conductance in hybrid three-terminal QD system is due to the nonequilibrium spin accumulation in the dot and has been explained in details in Ref. 8 .

In turn, in the antiparallel magnetic configuration (Fig. 5) the differential conductance $G_{S}^{A P}$ behaves similarly to that one obtained for $p=0$ because total population of electrons with a given spin direction in both leads remains constant disregarding of the value of $p$.

On the other hand, current cross-correlations exhibit both qualitative and quantitative differences in both magnetic configurations comparing with $p=0$ case. First of all, the current cross-correlations become enhanced in the vicinity of particle-hole symmetry point for $e V>E_{++}^{A}$ and for $e V<E_{--}^{A}$ in both magnetic configurations (compare Figs.4, 5 with Fig. 2).

FIG. 5: (color online) Differential conductance $G_{S}$ (a) and corresponding current cross-correlations (b) as a function of bias voltage and detuning $\delta$ in antiparallel magnetic configuration AP calculated for spin polarization $p=0.5$. The other parameters are as in Fig. 2.

However, maximal positive values of $S_{L R}$ in the Palignment become suppressed, whereas negative values are slightly increased (in the sense of absolute values) which can be clearly seen in Fig. 4 . In turn, $S_{L R}$ in AP configuration differs significantly from that in $\mathrm{P}$ configuration and also from that in the nonmagnetic case. One can notice that current cross-correlations for antiparallel magnetic configuration reach larger maximal values comparing with $S_{L R}$ for both parallel alignment and $p=0$ case.

To provide a deeper insight into $p$ dependence of Andreev current $\left(I_{S}\right)$ and corresponding current crosscorrelation $S_{L R}$, in both magnetic configurations, we derive some approximative analytical formulas assuming low temperature limit. Here, we concentrate on the particle-hole symmetric case, i. e. $\delta \approx 0$ as for $\delta \neq 0$ the obtained formulas become cumbersome. The correspond- 
ing formulas for $\delta \approx 0$ and for $\frac{1}{2}\left[U-\Gamma_{S}\right] \lesssim e V \lesssim \frac{1}{2}\left[U+\Gamma_{S}\right]$ are:

$$
\begin{aligned}
I_{S}^{P} & =\frac{4\left(1-p^{2}\right)}{3+p^{2}} I_{0} \\
S_{L R}^{P} & =\frac{1+21 p^{2}-29 p^{4}+7 p^{6}}{2\left(3+p^{2}\right)^{3}} S_{0}
\end{aligned}
$$

for parallel $(P)$, and

$$
\begin{aligned}
I_{S}^{A P} & =\frac{4}{3} I_{0} \\
S_{L R}^{A P} & =\frac{1+9 p^{2}}{54} S_{0}
\end{aligned}
$$

for antiparallel (AP) alignment, where $I_{0}=e \Gamma / \hbar$. The analytical formulas for $S_{L R}$ at negative bias voltage $e V<$ 0 in the corresponding regions are the same as for $e V>0$, whereas that for current are obtained from the relation, $I_{S}(\delta, e V)=-I_{S}(-\delta,-e V)$.

The above formulas confirm the deduced $p$-dependence of current-current cross correlations. Specifically, $S_{L R}$ in parallel magnetic configuration changes nonmonotonically with increasing $p$. For small values of polarization factor $S_{L R}$ grows with increasing $p$ until achieving maximum at $p=\sqrt{1 / 23(27-8 \sqrt{6})} \approx 0.57$. It is worth noting that such a value of spin polarization is observed for typical ferromagnets. With further increase of $p, S_{L R}$ decreases with increasing $p$. Finally, for halfmetallic leads $(p=1)$ current-current cross-correlations vanish and so does the current. In turn, current crosscorrelations in AP alignment monotonically grow with increasing $p$ achieving maximum for half-metallic leads $(p=1)$.

The next step in the current, for $\delta \approx 0$, appears at $e V \gtrsim \frac{1}{2}\left[U+\Gamma_{S}\right]$. In this regime, in the parallel magnetic configuration, the current and the corresponding current cross-correlations are described by the following formulas:

$$
\begin{aligned}
I_{S}^{P} & =2\left(1-p^{2}\right) I_{0} \\
S_{L R}^{P} & =\frac{p^{4}\left(1-p^{2}\right)}{4} S_{0} .
\end{aligned}
$$

The above formula shows that $S_{L R}$ is non-negative for all values of polarization factor and nonmonotonically depends on $p$ achieving maximum at $p=1 / \sqrt{2}$. In AP alignment current becomes independent from spin polarization factor $p$ and reaches its maximal value $I_{S}^{A P}=2 I_{0}$, while for the current-current cross-correlations one finds $S_{L R}^{A P}=p^{2} / 4$. Increase of $S_{L R}^{A P}$ is a result of enhanced CAR processes at the expense of DAR processes, which become suppressed while increasing $p$.

Moreover, an applied magnetic field influences current and corresponding current cross-correlations. At the first step in the current one finds the following formulas for $I_{S}$ and $S_{L R}$ calculated for $B / U=0.5$ :

$$
\begin{aligned}
I_{S}^{P} & =\frac{(p-1)(p+1)(p+3)}{2} I_{0} \\
S_{L R}^{P} & =\frac{(1-p)(1+p)\left(2-10 p+5 p^{2}+12 p^{3}+3 p^{4}\right)}{64} S_{0} .
\end{aligned}
$$

in $\mathrm{P}$ configuration, and

$$
\begin{aligned}
I_{S}^{A P} & =\frac{3}{2} I_{0} \\
S_{L R}^{A P} & =\frac{2+13 p^{2}}{64} S_{0},
\end{aligned}
$$

in $\mathrm{A} P$ configuration. Accordingly, for bias voltages at which the second step in the current occurs both $I_{S}$ and $S_{L R}$ are not influenced by magnetic field and resulting formulas remain the same as in the absence of magnetic field.

Finally, in the case of half-metallic leads in AP alignment current-current cross-correlations acquire nonnegative values in the whole range of bias voltage and detuning parameter $\delta$ (see Fig. 6). This is a result of total blockage of tunneling processes through the left and right junctions in opposite directions, as there are no available states in a given ferromagnetic lead for electrons incoming to the other ferromagnetic electrode. Moreover, both current and current-current cross-correlations become maximized. Maximization of current cross-correlations is well understood as for half-metallic leads in the AP alignment only CAR processes can contribute to Andreev current, whereas DAR processes are totally blocked, since in given electrode only electrons with one spin orientation are available. From the experimental point of view, such a device can be used for verification of the presence and role of CAR processes. Although, ideal halfmetallic leads are rather unaccessible in real systems, one can utilize the change in magnetic configuration of magnetic electrodes from antiparallel to parallel alignment in order to quantify the role of CAR comparing to DAR. Such protocol can be used because any switch of magnetic alignment of the system affects only CAR, whereas 


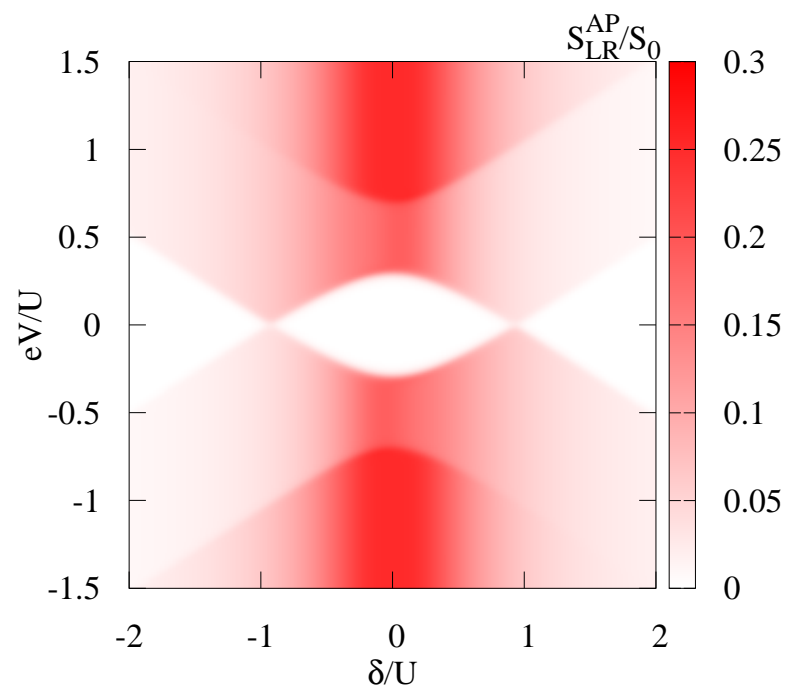

FIG. 6: (color online) Current cross-correlations as a function of bias voltage and detuning $\delta$ in antiparallel magnetic configuration $A P$ calculated for spin polarization $p=1$. The other parameters are as in Fig. 2.

DAR processes are not influenced. By comparing the transport properties of the system in both magnetic configurations, some information on CAR can be extracted.

\section{CONCLUSIONS}

In this paper we have analyzed the current crosscorrelations and differential conductance corresponding to the Andreev current flowing in single quantum dot based Cooper pair splitters. We have shown that finite magnetic field leads to the splitting of Andreev bound states, which can be clearly seen in the differential conductance characteristics. The current cross-correlations exhibit both positive and negative values in zero and finite magnetic field. However, finite magnetic field tends to suppress negative values (in the sense of absolute value) of the current cross-correlations and simultaneously it enhances the positive values of this quantity at least in specified regions of transport parameters. Moreover, we have found that in the vicinity of particle-hole symmetry point the current cross-correlations are suppressed to zero and the splitting pairs of electrons ending in two separate normal metal leads become uncorrelated. However, using ferromagnetic leads, in place of nonmagnetic ones, can restore entanglement of pair of split electrons in this transport regime. This finding is important as one wants to obtain large current of entanglement split pairs of electrons and as the Andreev current achieves maximum values in the particle-hole symmetry point.

When external weakly coupled leads are ferromagnetic the behavior of transport properties become more interesting. Especially, we have found negative differential conductance in the parallel magnetic configuration. Moreover, significantly different behavior of current cross-correlations has been found in both magnetic configurations compared to the nonmagnetic case. Furthermore, the dependence of current cross-correlations on leads' polarization factor $p$ has been addressed for both magnetic alignments. Specifically, in the parallel magnetic configuration the current-current cross-correlations exhibit nonmonotonic behavior with varying $p$; they reach maximal value for finite polarization factor suggesting that competition between CAR and DAR processes in $\mathrm{P}$ alignment is not trivial. On the other hand, in the antiparallel magnetic configuration contribution of CAR processes gradually grows with increasing $p$, and finally for half-metallic leads $p=1$, CAR becomes the only source of Andreev current. We have also indicated possible experimental utilization of the considered system in order to quantify the different processes contributed to the transport.

\section{Acknowledgments}

K.W. acknowledges support from the Polish National Science Centre from funds awarded through the decision No. DEC-2013/10/E/ST3/00213. The authors thank I. Weymann for discussions.

\section{REFERENCES}

* Electronic address: ptrocha@amu.edu.pl

1 Martin-Rodero A and Levy Yeyati A 2011 Adv. Phys. 60:6 899

2 Futterer D, Governale M, Pala M G and König J 2009 Phys. Rev. B 79054505

3 Sothmann B, Futterer D, Governale M and König J 2010 Phys. Rev. B 82094514

4 Eldridge J, Pala M G, Governale M and König J 2010 Phys. Rev. B 82184507

5 Hofstetter L, Geresdi A, Aagesen M, Nygård
J, Schönenberger C and Csonka S 2010 Phys. Rev. Lett. 104246804

${ }^{6}$ Sadovskyy I A, Lesovik G B, Blatter G, Jonckheere T and Martin T 2012 Phys. Rev. B 85125442

7 Wójcik K P and Weymann I 2014 Phys. Rev. B 89165303

8 Weymann I and Trocha P 2014 Phys. Rev. B 89115305

9 Trocha P and Barnaś J 2014 Phys. Rev. B 89245418

10 Trocha P and Weymann I 2015 Phys. Rev. B 91235424

11 Feinberg D, Jonckheere T, Rech J, Martin T, Douçot B and Mélin R 2015 Eur. Phys. J. B 8899 
12 Mélin R, Sotto M, Feinberg D, Caputo J -G and Douçot B 2016 Phys. Rev. B 93115436

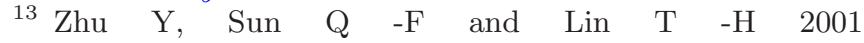
Phys. Rev. B 65024516

14 Sauret O, Feinberg D and Martin T 2004 Phys. Rev. B 70245313

15 Chen Z, Wang B, Xing D Y and Wang J 2004 Appl. Phys. Lett. 852553

16 Feng J -F, Wua X $-\mathrm{S}$ and Jiang S J. Appl. Phys. 99 08F713

17 Golubev D S and Zaikin A D 2007 Phys. Rev. B 76184510

18 Hofstetter L, Csonka S, Nygård J and Schönenberger C 2010 Nature 461960

19 Herrmann L G, Portier F, Roche P, Levy Yeyati A, Kontos T and Strunk C 2010 Phys. Rev. Lett. 104026801

${ }^{20}$ Hofstetter L, Csonka S, Baumgartner A, Fülöp G, d'Hollosy S, Nygård J and Schönenberger C 2011 Phys. Rev. Lett. 107136801

${ }^{21}$ de Franceschi S, Kouwenhoven L P, Schönenberger C and Wernsdorfer W 2011 Nat. Nanotech. 5703

22 Andreev A F 1964 Zh. Eksp. Teor. Fiz. 461823 [Sov. Phys. JETP 19 1228]

23 Chevallier D, Rech J, Jonckheere T and Martin T 2011 Phys. Rev. B 83125421

${ }^{24}$ Rech J, Chevallier D, Jonckheere T and Martin T 2012 Phys. Rev. B 85035419

25 Blanter Ya M and Büttiker M 2000 Phys. Rep. 336 1-166

26 Sukhorukov E V, Jordan A N, Gustavsson S, Leturcq R, Ihn T and Ensslin K 2007 Nat. Phys. 3243

27 McClure D T, DiCarlo L, Zhang Y, Engel H -A, Marcus C M, Hanson M P and Gossard A C 2007 Phys. Rev. Lett. 98056801

${ }^{28}$ Das A, Ronen Y, Heiblum M, Mahalu D, Kretinin A V and Shtrikman H 2012 Nat. Commun. 31165

29 Martin T 1996 Phys. Lett. A 220137

30 Torrès J and Martin T 1999 Eur. Phys. J. B 12319

31 Lesovik G B, Martin T and Blatter G 2001 Eur. Phys. J. B 24287

32 Bignon G, Houzet M, Pistolesi F and Hekking F W J 2004 Europhys. Lett. 67110
33 Mélin R, Benjamin C and Martin T 2008 Phys. Rev. B 77094512

34 Dong B and Lei X L 2009 Phys. Rev. B 80153305

35 Freyn A, Flöser M and Mélin R 2010 Phys. Rev. B 82014510

36 Freyn A, Douçot B, Feinberg D and Mélin R 2011 Phys. Rev. Lett. 106257005

37 Riwar R -P, Badiane D M, Houzet M, Meyer J S, Nazarov Y V 2016 Physica E 76231

38 Wrześniewski K, Trocha P and Weymann I 2017 J. Phys.: Cond. Matter 29195302

39 Henny M, Oberholzer S, Strunk C, Heinzel T, Ensslin K, Holland M, Schönenberger C 1999 Science 284296

40 Oliver W D, Kim J, Liu R C, Yamamoto Y 1999 Science $\mathbf{2 8 4} 299$

41 Bagrets D A and Nazarov Y W 2003 Phys. Rev. B 67085316

42 Cottet A, Belzig W, and Bruder C 2004 Phys. Rev. Lett. 92206801

43 Chtchelkatchev N M, Blatter G, Lesovik G B and Martin T 2002 Phys. Rev. B 66161320

44 Sauret O, Martin T and Feinberg D 2005 Phys. Rev. B 72024544

45 Flöser M, Feinberg D and Mélin R 2013 Phys. Rev. B 88094517

46 Rozhkov A V and Arovas D P 2000 Phys. Rev. B 626687

47 Schoeller H and Schön G 1994 Phys. Rev. B 50 18436; König J, Schmid J, Schoeller H and Schön G 1996 Phys. Rev. B 5416820

48 Thielmann A, Hettler M H, König J and Schön G 2005 Phys. Rev. Lett. 95146806

49 Governale M, Pala M G and König J 2008 Phys. Rev. B 77134513

50 Braggio A, Governale M, Pala M G, and König J, Solid State Commun. 2011151155

51 Kłobus W, Grudka A, Baumgartner A, Tomaszewski D, Schönenberger C, and Martinek J 2014 Phys. Rev. B 89125404 